\title{
Ellos tienen las leyes; nosotras, el misoprostol
}

They Have Laws, We Have the Misoprostol

Fecha de recepción: 06/09/2021

Fecha de aceptación: 26/11/2021

Fecha de publicación: 07/03/2022

https://doi.org/10.48102/if.2022.v2.n1.196

Marea Verde Tabasco*

mareaverdetabasco@gmail.com

ORCID: https://orcid.org/0000-0002-4308-9303

Marea Verde Tabasco

México

* Las integrantes de la colectiva Marea Verde Tabasco iniciaron su activismo formalmente el 28 de septiembre de 2019, cuando organizaron la primera marcha en el estado de Tabasco con motivo del Día de Acción Global por el acceso al Aborto Legal y Seguro; desde entonces, se han dedicado a compartir contenido en sus redes sociales para desmitificar el aborto, han participado en conversatorios y han organizado talleres con el fin de informar sobre los derechos sexuales y reproductivos de las mujeres, además de brindar acompañamientos virtuales a mujeres con embarazos no deseados. 


\section{Resumen}

La criminalización de las mujeres que abortan ha sido el flagelo que las instituciones gubernamentales y religiosas han impuesto sobre quienes deciden abortar y sobre las personas que acompañan o instrumentan este procedimiento. ${ }^{1}$ A lo largo de la historia, han existido tímidas iniciativas de ley para despenalizarlo, aunque solamente en Ciudad de México, Oaxaca, Hidalgo y recientemente Veracruz ${ }^{2}$ han sido llevadas al pleno y votadas en favor de las mujeres. Ante la misoginia del Estado, las feministas encontramos, en el acompañamiento a mujeres que necesitan abortar, una fuente de sororidad. En Tabasco, la situación no es distinta, por lo que las feministas hemos exigido, a través de nuestro activismo, que las mujeres dejen de ser criminalizadas por ejercer su autonomía reproductiva.

\section{Palabras clave}

Aborto, acompañamiento, criminalización, feminismo, legislación.

\section{Abstract}

The criminalization of women who have abortions has been the scourge that governmental and religious institutions have imposed on those who decide to have abortions and on the people who accompany or perform this procedure. ${ }^{3}$

1 Este ensayo fue entregado para su dictaminación el 6 de septiembre de 2021; no contábamos con que, al día siguiente, la Suprema Corte de Justicia de la Nación (SCJN) emitiría la acción de inconstitucionalidad para el delito de aborto establecido en el Código Penal de Coahuila, lo que declaró la descriminalización en el estado y estableció un precedente que generó jurisprudencia para los demás estados de la República. Cabe precisar que, en Tabasco, aun cuando tenemos este referente, las leyes locales no han sido armonizadas, por lo que las mujeres que abortan y quienes las acompañamos corremos el riesgo de ser sujetas a proceso penal. En el supuesto de que una mujer fuera criminalizada, se tendría que recurrir a un amparo y el o la jueza estaría obligada a observar el precedente de la SCJN mientras no se armonicen las leyes locales.

2 Al momento de entregar este ensayo para su revisión, sólo cuatro entidades habían legislado a favor de la despenalización del aborto. Posteriormente, el Congreso de Baja California legisló en favor de la despenalización hasta las doce semanas de gestación, convirtiéndose en la quinta entidad que emite un fallo en favor de los derechos reproductivos de las mujeres. 3 This essay was submitted for review on September 6, 2021; we did not count on the fact that, the following day, the Supreme Court of Justice of the Nation (SCJN) would issue the action of unconstitutionality for the crime of abortion established in the Penal Code of Coahuila, which declared decriminalization in the state and established a precedent that generated jurisprudence for the other states of the Republic. It should be noted that in

Marea Verde Tabasco (2022). Ellos tienen las leyes; nosotras, el misoprostol. Iberoforum, Revista de Ciencias Sociales, Nueva Época, 2(1), 1-18, Ensayos, e000196. https://doi.org/10.48102/if.2022.v2.n1.196

Licencia Pública Internacional - CC BY-NC-ND 4.0 
Throughout history, there have been timid rights of initiative to decriminalize abortion, although only in Mexico City, Oaxaca, Hidalgo and recently Veracruz ${ }^{4}$ have they been brought to the plenary and voted in favor of women. In the face of the misogyny of the State, feminists find a source of sisterhood in accompanying women in need of abortion. In Tabasco, the situation is no different, and feminists have demanded, through our activism, that women no longer be criminalized for exercising their reproductive autonomy.

Keywords

Abortion, accompaniment, criminalization, feminism, legislation.

Tabasco, even though we have this reference, local laws have not been harmonized, so that women who have abortions and those who accompany them run the risk of being subject to criminal prosecution. In the event that a woman is criminalized, she would have to resort to an amparo and the judge would be obliged to observe the SCJN precedent until local laws are harmonized.

4 When this essay was submitted for review, only four states had legislated in favor of decriminalizing abortion. Subsequently, the Congress of Baja California legislated in favor of decriminalization up to twelve weeks of gestation, becoming the fifth state to issue a ruling in favor of women's reproductive rights. 
Dedicamos este ensayo a la sororidad entre

mujeres, ${ }^{5}$ que nos ha salvado de situaciones

de las que no creíamos salir.

\section{Introducción}

El himno feminista titulado El violador eres tú, escrito por la colectiva chilena Las Tesis, señala en su primera estrofa: "El patriarcado es un juez/ que nos juzga por nacer,/ y nuestro castigo es/ la violencia que no ves". En este ensayo, hablaremos de cómo el patriarcado ${ }^{6}$ ha juzgado a las mujeres y cómo el Estado ha criminalizado a las que deciden sobre sus cuerpos mediante la interrupción de un embarazo no deseado. ${ }^{7}$ Desde el feminismo y como activistas, hemos decidido convertirnos en "acompañantas"; 8 desde la sororidad, abrazamos y acompañamos a toda mujer que decide sobre su cuerpo; lo hacemos fuera del dogma médico y en la frontera de la legalidad, desafiando al Estado opresor (Guerra, 2019). Este ensayo está basado en nuestra visión de mujeres acompañantas, que es la experiencia feminista abortera; la perspectiva de mujeres que sienten empatía por otra mujer.

5 Sororidad. Del latín soror, que significa "hermandad". En México, quien introdujo esta palabra fue la antropóloga feminista Marcela Lagarde, quien la define como "una dimensión ética, política y práctica del feminismo contemporáneo. Es una experiencia de las mujeres que conduce a la búsqueda de relaciones positivas y a la alianza existencial y política, cuerpo a cuerpo, subjetividad a subjetividad con otras mujeres, para contribuir con acciones específicas a la eliminación social de todas las formas de opresión y al apoyo mutuo para lograr el poderío genérico de todas y el empoderamiento vital de cada mujer" (Lagarde, 2006, p. 126). 6 La definición resumida de "patriarcado" de Gerda Lerner (1986) es un "sistema social en el que los hombres tienen el poder primario y predominan en roles de liderazgo político, autoridad moral, privilegio social y control de la propiedad".

7 Según la solicitud de información al Tribunal Superior de Justicia de Tabasco (2020), generada a través del portal Infomex, sólo una persona ha sido sentenciada por el delito de aborto entre 2000 y 2020, pero no se especifica su sexo. En lo que va del 2021, existen cuatro carpetas de investigación iniciadas por el delito de aborto de acuerdo con el Secretariado Ejecutivo del Sistema Nacional de Seguridad Pública (2021).

8 Una acompañanta es aquella mujer que apoya y asesora a otra, brindándole información segura, confiable y científica sobre el aborto voluntario con medicamentos o mediante la aspiración manual endouterina (AMEU). Las acompañantas de aborto ayudan a conseguir los medicamentos necesarios para una interrupción. También asesoran a las mujeres y las acompañan durante el procedimiento para evaluar sus síntomas y estado de ánimo. Después, tienen comunicación una vez a la semana hasta que se cumpla un mes y se confirme que el aborto no tuvo complicaciones (Olvera et al., 2021; Morales, 2019).

Marea Verde Tabasco (2022). Ellos tienen las leyes; nosotras, el misoprostol. Iberoforum, Revista de Ciencias Sociales, Nueva Época, 2(1), 1-18, Ensayos, e000196. https://doi.org/10.48102/if.2022.v2.n1.196

Licencia Pública Internacional - CC BY-NC-ND 4.0 
Antes de comenzar a exponer la necesidad de descriminalizar el aborto y el acompañamiento, debemos tener claro quiénes conforman el sistema patriarcal: "son los jueces, el Estado, el presidente", siguiendo con otra estrofa del himno mencionado al inicio, pero también la sociedad, la iglesia y la familia. Todos estos elementos tienen una contribución clave a la violencia ejercida en contra de las mujeres que deciden sobre su vida y sus cuerpos.

La maternidad no debe ser impuesta por un sistema político; sin embargo, esta imposición se da, por ejemplo, a través de la legislación que criminaliza a las mujeres y no les garantiza el acceso a servicios de salud de calidad para interrumpir una gestación. Actualmente, la legislación establecida en el Código Penal y la Ley de Salud no garantiza condiciones justas para atender las problemáticas sociales de las mujeres, adolescentes y niñas en el estado de Tabasco; es una legislación obsoleta para el contexto en el que vivimos. Por otra parte, el sector salud, tanto público como privado, dogmatiza la práctica médica y niega el servicio de interrupción aun cuando la solicitante afirme haber sido violada, con lo que se ignora deliberadamente la Norma Oficial Mexicana NOM-046-SSA2-2005. ${ }^{9}$

En México hay dos antecedentes clave para la despenalización legal y la descriminalización social de las mujeres que abortan, ${ }^{10}$ los cuales generaron las circunstancias idóneas para que el Estado reconozca que la decisión de ser madre sólo compete a la mujer en su esfera privada y no es facultad del Estado inmiscuirse. El primero data de 1931, cuando se despenalizó el aborto en el Código Penal para el Distrito Federal bajo tres causales: por violación, cuando esté en peligro la vida de la mujer gestante y debido a una imprudencia de la mujer (Cano, 1990). Esta última resulta revictimizante pero, considerando el año en el que se emitió, es un precedente

9 La NOM-046-SSA2-2005 (Violencia familiar, sexual y contra las mujeres. Criterios para la prevención y atención) establece que los casos de violación sexual son urgencias médicas y requieren atención inmediata; además, las instituciones de salud deben prestar el servicio de interrupción voluntaria del embarazo en estos casos (Secretaría de Salud, 2009).

10 La publicación del fallo de la SCJN del 7 de septiembre de 2021 que descriminaliza a nivel nacional el delito de aborto, sin especificar hasta las cuántas semanas de gestación, no significa que se haya despenalizado en todas las entidades que aún no legislan al respecto pues, a diferencia de la despenalización - que significa la abolición de las penas criminales en relación con el acto-, entendemos como descriminalización también el reflejo del cambio en los puntos de vista sociales y morales. 
que reconoce que hay circunstancias en las que las mujeres que están gestando necesitan interrumpir la gestación bajo cualquier causa.

El segundo antecedente se dio en 1936, cuando la doctora Matilde Rodríguez Cabo leyó en la Convención de Unificación Penal la ponencia "Aborto por causas legales y económicas", escrita por la doctora Ofelia Domínguez Navarro, en la que solicitó que se derogaran los artículos que penalizaban esta práctica (Cano, 1988). El documento expuesto es resultado del viaje de investigación que hizo la doctora Domínguez en 1929 a la entonces Unión de Repúblicas Socialistas Soviéticas (URSS). Durante su estancia en ese país otrora segunda potencia mundial, en donde ya se reconocían a las mujeres algunos derechos que en México se harían patentes años después, analizó la protección para la madre y las infancias desde la perspectiva socialista. Cabe mencionar que en aquella nación se legisló el aborto voluntario desde 1922. La observación, análisis, desarrollo y propuestas que realizó Domínguez Navarro parten de una visión sociológica y legal; la autora enfatiza que las carencias económicas son un factor que se debe considerar en la decisión de una mujer de interrumpir una gestación (Cano, 1990).

En 1979, el Partido Comunista Mexicano presentó por primera vez la Ley para Despenalizar el Aborto (Cano, 1990), inspirada en los estudios de la doctora Domínguez Navarro. Este instituto político fue avasallado por el partido hegemónico en el poder y la iniciativa no llegó a consolidarse, pero marcó el inicio de un debate que se sostiene hasta la actualidad en las entidades de la República.

\section{Antecedentes de la despenalización del aborto en las entidades federativas de México}

Los debates y luchas por despenalizar este derecho humano se trasladaron entonces hacia los estados, como fue el caso de Chiapas en la década de $1990^{11}$ y Baja California en el 2000,12 en donde se practicó la primera in-

11 Iniciativa con proyecto de decreto por el que se reforman y adicionan diversas disposiciones del Código Penal Federal, del Código Federal de Procedimientos Penales y de la Ley General de Salud, en materia de aborto legalizado, a cargo del diputado Nazario Norberto Sánchez del grupo parlamentario del Partido de la Revolución Democrática (Senado de la República, 2011). En la exposición de motivos, se narran cronológicamente los antecedentes de la despenalización del aborto en México. La iniciativa fue publicada en la Gaceta de la Comisión Permanente del Senado de la República.

12 El pasado 29 de octubre de 2021, el Congreso de Baja California legisló en favor de la despenalización del aborto, lo que convirtió a esta entidad en el quinto estado que lo hace 
terrupción voluntaria en un caso por violación. Los debates fueron constantes porque, cada vez que algún orden de gobierno, ya sea ejecutivo o legislativo, presentó alguna iniciativa al respecto, hubo presión y activismo por parte del Partido Acción Nacional (PAN). Aunque el PAN no contaba más que con una minoría de representación en el Congreso, fue apoyado por grupos conservadores y religiosos que poseían enormes cotos de poder. De ahí que sólo quedaron en debate esos intentos por restituir a las mujeres la potestad sobre sus cuerpos.

Pese a que el PAN logró la presidencia de la República en el 2000, el gobierno local del entonces Distrito Federal presentó la iniciativa que la Asamblea Legislativa aprobó; desde el año 2007, se despenalizó la interrupción del embarazo sin causal hasta las doce semanas de gestación. Este logro para los derechos de las mujeres fue posible gracias a que desde 1997, en lo que ahora es la Ciudad de México, han gobernado localmente los partidos de izquierda inspirados en la justicia reproductiva que los países socialistas llevaron a cabo en las décadas anteriores, a diferencia del partido en la presidencia de la República, de clara tendencia ideológica conservadora.

En el caso de Tabasco - cuyos gobiernos mayoritariamente priistas, luego perredistas y actualmente morenistas han mostrado una ideología de izquierda-, no se ha podido lograr la despenalización debido a que, sin importar la ideología partidista, quienes han integrado los gobiernos han prestado atención a los grupos conservadores - como la Iglesia católicaen detrimento de los derechos de las mujeres. Además de servir a los intereses de la Iglesia, se han enfocado en mantener a la mujer en el espacio privado, sin que ésta tenga la posibilidad de decidir sobre su cuerpo y en general sobre todas las esferas de su vida, pues se atribuye la potestad de las mujeres al esposo o al padre según sea el caso. Su propio machismo ha estado reflejado en su forma de gobernar o legislar. Esta indiferencia manifestada frente a las voces de las feministas tabasqueñas es un claro ejemplo de la misoginia en el poder, tema que abordaremos más adelante.

Los debates siguieron dándose en los congresos locales. Fue hasta 2019 que el Congreso de Oaxaca legisló en favor de los derechos reproductivos de las mujeres y despenalizó el aborto por cualquier causal hasta las

en el país; sin embargo, tomamos de referencia el intento del año 2000 como antecedente de la lucha por la defensa de los derechos de las mujeres. 
doce semanas de gestación. El 30 de junio de 2021, el Congreso hidalguense votó la iniciativa con proyecto de decreto, en el que se reformaron los artículos 154 al 158 del Código Penal del Estado de Hidalgo; el Congreso hizo su parte y descriminalizó la interrupción del embarazo también hasta las doce semanas de gestación; no obstante, fue muy incisivo al imponer sanciones que van de seis meses a un año de prisión y una multa de entre diez y cuarenta días para la mujer que se practique un aborto $-\mathrm{o}$ a quien lo facilite- después de las doce semanas de iniciada la gestación (Ginecea, 2021; Poder Ejecutivo del Estado de Hidalgo, 2021). Caso contrario: el 20 de julio de 2021, el Congreso veracruzano aprobó sin tanto debate la iniciativa que reforma los artículos 149, 150, 151, 153 y 154 y deroga el artículo 152, todos del Código Penal para el Estado de Veracruz; es notable la diferencia en las penas otorgadas a quien rebase el límite de las doce semanas: de quince días a dos meses de tratamiento en libertad, entendiendo esto último como la instrumentación de medidas integrales de salud con respeto a los derechos humanos (Congreso del Estado de Veracruz, 2021).

Éstos son los únicos estados de nuestro país -CDMX, Oaxaca, Veracruz e Hidalgo $-{ }^{13}$ en donde las mujeres tienen garantía plena de no ser criminalizadas por ejercer el derecho a decidir sobre su cuerpo. Antes de la organización de las colectivas aborteras -entre ellas, nuestra colectiva, Marea Verde Tabasco-, las mujeres que decidían interrumpir su embarazo no contaban con el apoyo del Estado, dada la precariedad del sistema de salud mexicano. Por ejemplo, en Tabasco, el acceso a un aborto seguro no ha sido del todo garantizado pues, aun cuando desde hace diez años se cuenta con el servicio de aspiración manual endouterina (AMEU), técnica implementada para abortos seguros, el acceso a éste ha sido burocratizado y restrictivo, incluso para las excluyentes de responsabilidad establecidas en el Código Penal Estatal.

Las autoridades han ido más lejos: tuvieron casi oculta la existencia de la AMEU y no hay estadística de cuántas interrupciones se realizaron por medio de la AMEU, de la técnica de legrado uterino instrumentado (LUI) o del uso de medicamento como mifepristona y misoprostol; dado que los

13 Como hemos mencionado, este ensayo fue entregado para su revisión un día antes de la descriminalización que emitió la SCJN y un mes después de que el Congreso de Baja California despenalizara el aborto. 
datos que se registraron no distinguen entre las tres técnicas, también se perdió esta valiosa estadística (Anónimo, comunicación personal, 28 de octubre de 2021). ${ }^{14}$

En los estados en donde todavía no se encuentra despenalizado el aborto voluntario, se ha podido tener acceso a éste en el sector privado de salud -hospitales, consultorios o médicos-. A nuestra colectiva, han llegado testimonios de chicas que han sido revictimizadas por personal de salud de dichas clínicas privadas legalmente constituidas; alegando escenarios que ponen en peligro la vida de la mujer gestante, cobran hasta diez mil pesos para llevar a cabo el aborto; son profesionales que lucran con la necesidad de las mujeres; esto también resulta discriminatorio puesto que el procedimiento no es asequible para las mujeres en situación de vulnerabilidad económica. En este contexto, aplica la frase "Las ricas abortan, las pobres mueren". Estas últimas han tenido que recurrir a la clandestinidad, en donde las condiciones pueden poner en riesgo su vida. Es una realidad dolorosa que no debemos ocultar.

\section{Dogmas y falacias en torno al aborto}

Los prejuicios que se generan en torno al aborto son de toda índole; destacan dos aspectos: lo social y lo dogmático religioso.

Dentro de los prejuicios sociales, encontramos falacias como la depresión postaborto; un trastorno sin sustento científico pues, de acuerdo con las conclusiones de una investigación publicada en la revista de la Asociación Americana de Psicología (APA por sus siglas en inglés), falta evidencia que demuestre que las mujeres que interrumpen el embarazo de manera voluntaria sufran en consecuencia un trastorno de salud mental (Major et al., 2009).

Otra falacia en torno a la interrupción voluntaria del embarazo es la condenación que la sociedad hace de la mujer por ejercer su libertad sexual: se le obliga a maternar, aunque ella no lo desee, como "castigo" por su lascivia; lo que se refleja en frases como: ahora lo tienes aunque no lo quieras, para qué abres las piernas, eso te pasa por caliente, y un doloroso etcétera.

14 Información obtenida en una entrevista confidencial con una aliada empleada de la Secretaría de Salud de Tabasco. Los datos pueden ser constatados en el sitio web del Subsistema Automatizado de Egresos Hospitalarios (SAEH). 
Ésta es una de las más aberrantes falacias, ya que es muy frecuente que sea la misma familia la que se yerga como juez y verdugo. El impacto emocional que representa el rechazo de la familia resulta cruel por ser supuestamente ésta el núcleo de la sociedad, la célula básica en la que se sustenta la nación. El espacio que debería ser un refugio seguro para todas, al verse comprometido, deja en el abandono emocional y en indefensión a una mujer que no desea gestar; por lo tanto, a ésta no le queda otra opción más que recurrir a la clandestinidad.

Otra serie de falacias es que el aborto provoca esterilidad o que el procedimiento causa hemorragia incontrolable hasta ocasionar la muerte, aunque se realice en un hospital con todos los cuidados de salud: "El personal de salud en general coincide en señalar que el aborto practicado en condiciones inadecuadas provoca elevada morbi-mortalidad que se traduce en un grave problema de salud pública y de la sociedad" (Ballinas, 2014, p. 8). Sin embargo, diversas organizaciones de salud han publicado protocolos para la interrupción segura en casa con medicamentos; entre ellas, la Organización Mundial de la Salud [OMS] (2014), la Federación Internacional de Ginecología y Obstetricia [FIGO] (2017), Ipas México, el Consorcio Latinoamericano Contra el Aborto Inseguro [CLACAI] (s. f.), Ímpetu A. C. y Fondo María (2018).

En cuanto a los prejuicios derivados de los dogmas cristianos, podemos decir que los grupos religiosos, sean católicos o protestantes, justifican la posesión del cuerpo de las mujeres a partir de sus interpretaciones absolutas sobre la Biblia, a la que consideran una fuente fundamental. Con el pretexto de que es mandato divino, las mujeres no pueden decidir sobre su maternidad o la cantidad de hijos que desean. Esto no sólo cancela sus derechos reproductivos; también las criminaliza y las condena a sufrir "la ira de Dios" si contravienen estos designios. La postura de estos grupos religiosos en contra de las mujeres que deciden interrumpir su embarazo ha llegado más lejos al presentar reformas legislativas que van en contra de los derechos sexuales y reproductivos de las mujeres; un ejemplo de estas iniciativas es el "pin parental". ${ }^{15}$

15 Esta propuesta para reformar ordenamientos legales nació en España y es impulsada por el partido conservador Vox. En México, ha sido propuesta por partidos como Encuentro Social y Acción Nacional. Esta iniciativa trata de legislar que las escuelas no impartan a las niñas y niños educación sexual integral con perspectiva de género sin la autorización previa de sus padres. 


\section{Acceso al aborto seguro y legal en Tabasco}

En el estado de Tabasco, existen organizaciones dedicadas a realizar campañas mediáticas para perseguir a las mujeres que deciden interrumpir sus embarazos; un ejemplo de ello son las iniciativas impulsadas por el Consejo Interreligioso Local, el Colegio de Abogados Tabasqueños, el Comité Cívico Cultural de Tabasco y la Sociedad de Abogados J. Reuben Clark capítulo Tabasco, que presentó en 2018 una iniciativa de ley para "proteger" al no nacido. Esta iniciativa antecede a la impulsada por la entonces diputada Karla Rabelo de la fracción parlamentaria de MORENA, quien presentó el 21 de octubre de 2020, en el Pleno del Congreso local, una iniciativa de ley para reformar el artículo 2 de la Constitución Política del Estado Libre y Soberano de Tabasco; dicha propuesta buscaba "garantizar" el derecho a la vida del no nacido. Ambos documentos no fueron discutidos ni aprobados por la LXIII Legislatura que integraba el Congreso estatal al momento de ser presentados.

Estas iniciativas suelen estar disfrazadas de preocupación pero, en realidad, hacen poco o nada por las personas que ya alcanzaron el estatus de ciudadanía. Podemos pensar, por ejemplo, en aquéllas que no gozan de derechos básicos como una vivienda: de acuerdo a la encuesta de población y vivienda de 2020 realizada por el Instituto Nacional de Estadística y Geografía [INEGI] (2020a, 2020b), sólo $27.8 \%$ de la población en Tabasco cuenta con una vivienda propia; la misma encuesta revela que sólo $6.43 \%$ de la población económicamente activa cuenta con un empleo formal. Dado el aumento de la precariedad económica, esta clase de legisladoras y legisladores quedan como hipócritas que se preocupan por quien todavía es un embrión, pero se desentienden legalmente de quienes son poseedores de los derechos que la ciudadanía les otorga.

Tabasco posee uno de los códigos penales más restrictivos en materia de derechos reproductivos. Las causales para acceder a un aborto se limitan a casos de violación, cuando se encuentre en riesgo la vida de la mujer y por inseminación artificial no consensuada. Aun cuando estos son aspectos no punibles, las autoridades siguen criminalizando a las mujeres y les niegan la atención médica necesaria al anteponer sus prejuicios a la vida misma de la mujer; como ya se mencionó, no garantizan el acceso a la NOM-046, pues el personal médico aduce que el artículo 136 del Código Penal estatal no está homologado con dicha norma, ya que establece que se debe comprobar el delito de violación. 
En Tabasco, se han solicitado veinticinco sentencias por el delito de aborto y sus delitos relacionados como infanticidio y homicidio en razón de parentesco (Tribunal Superior de Justicia, 2020); ${ }^{16}$ de igual manera, se han iniciado diecisiete carpetas de investigación por el mismo delito del año 2015 a julio de 2021 (Secretariado Ejecutivo del Sistema Nacional de Seguridad Pública, 2021; Subsistema Automatizado de Egresos Hospitalarios, 2021). Si bien pueden parecer números muy bajos, esto no minimiza ni elimina la criminalización que las autoridades realizan hacia la autonomía reproductiva de las tabasqueñas. Ante ello, muchas optan por la clandestinidad, pues, saben que el sistema de salud les negará el servicio y que los encargados de impartir justicia las criminalizarán si sospechan que se indujeron un aborto, lo que las deja en la indefensión total y a su suerte.

La ceguera de las autoridades ante esta problemática ha llevado a las mujeres a buscar alternativas para terminar con un embarazo no deseado sin poner en riesgo su vida, pues, aun cuando es delito abortar por voluntad, no tiene por qué ser inseguro. Las mujeres que buscan interrumpir una gestación no deseada han recurrido a los manuales emitidos por la OMS, FIGO e Ipas - una organización sin fines de lucro- para obtener información referente al suministro de misoprostol para expulsar un feto.

Las feministas, a través de las redes sociales, hemos resuelto el problema de atención a estas mujeres mediante grupos de apoyo y asesoría que brindan contención emocional, legal y logística para que puedan acceder a un aborto seguro.

\section{Respuesta feminista a la persecución del Estado: acompañamiento}

La violencia obstétrica que sufren frecuentemente las mujeres en los hospitales públicos, especialmente en el área de partos, es harto conocida. El personal de salud, médicas, médicos, enfermeras y enfermeros se yerguen como figuras de autoridad frente a sus pacientes, muchas veces como resultado del poder que la estructura patriarcal les ha conferido. Sabemos que no son todos los casos - no pretendemos generalizar-; sin

16 Información obtenida mediante el oficio TSJ/DEIC/426/2020, con fecha del 5 de octubre de 2020, a través de una solicitud de información al Tribunal Superior de Justicia del Estado de Tabasco realizada en la plataforma Infomex. 
embargo, la formación académica y sus conocimientos les conceden poder y contribuyen a naturalizar el trato déspota y violento hacia las pacientes (Ballinas, 2014).

En el lado opuesto, nos ubicamos las acompañantas aborteras. El feminismo nos ha sensibilizado para ejercer un buen trato hacia las mujeres que deciden abortar. Escuchamos sin juzgar, atendemos las emociones desde la empatía. El enfoque feminista para ver la vida es como la metáfora de las gafas violetas: nuestra visión del mundo ahora coloca a "las mujeres al frente y nuestros derechos al centro", frase dicha por la feminista Yndira Sandoval en la inauguración de la Escuela de Verano Feminista 2021 de Las Constituyentes y que se ha convertido en un lema que nos recuerda que somos nuestras sujetas políticas.

Por eso y porque somos conscientes de nuestras propias violencias, decidimos capacitarnos por nuestra cuenta. Contamos con el manual de práctica clínica para un aborto seguro de la OMS (2014), que es fácilmente localizable en la web; hacemos talleres, compartimos experiencias y vamos elaborando nuestros propios protocolos de acompañamiento a distancia.

Nos organizamos en grupos virtuales conformados por una acompañanta -quien se encarga de dirigir el procedimiento-, una supervisora -que cuenta con mayor experiencia y vigila el buen desempeño de la acompañanta-, una observadora -quien está en formación abortera- y la acompañada. Como parte de nuestro protocolo de primer contacto, realizamos una llamada telefónica a la mujer que necesita nuestro apoyo. La escuchamos atentamente y le explicamos detenidamente en qué consiste la interrupción con misoprostol; le indicamos la dieta y los cuidados necesarios al momento de realizar el procedimiento; también le damos palabras de aliento para hacerle sentir que no está sola.

Es muy triste reconocer que la mayoría de las mujeres que recurren a nosotras lo hacen cargadas de culpa, originada por esa idea patriarcal sembrada desde la infancia de que no somos dueñas de nuestros cuerpos. Es por eso por lo que damos tanta importancia al discurso que manejamos desde el primer contacto. No juzgamos; escuchamos, apoyamos y abrazamos desde la sororidad.

Este sentimiento de culpa y temor a ser juzgadas que impera en las mujeres que deciden sobre su cuerpo no es exclusivo de ellas o de las demás mujeres. Es una creencia generalizada entre la población. Las colecti- 
vas feministas Marea Verde Tabasco y Esmeralda realizamos una encuesta en 2020 en el territorio tabasqueño: según los resultados, $66 \%$ de los y las tabasqueñas conocen a alguien que ha abortado o han tenido un aborto voluntario; $73.6 \%$ conoce a alguien que pensó en abortar; no obstante, 61.9 \% indicó que no hay razones para acceder a un aborto. Esta información nos revela la disyuntiva social que existe cuando se trata de derechos reproductivos de las mujeres: por un lado, saben qué ocurre, conocen de cerca la realidad de las mujeres en situación de embarazo no deseado; pero, por otro lado, las obligan a llevar un proceso de gestación sin importar las consecuencias.

Hablar de aborto en Tabasco ha sido tan común que en todas las épocas se ha sabido quién lo practica, cuál comadrona o médico y dónde. La hipocresía de los grupos religiosos nunca ha sido un obstáculo para que esta información sea pública; incluso, ellos mismos recurren a los servicios clandestinos de estas personas con tal de terminar con el embarazo no deseado de alguna de sus familiares. Las feministas tenemos muy presente que lo personal es político. Hemos conocido casos de mujeres que han sido obligadas, por el hombre que las embarazó, a abortar en condiciones insalubres y violentas. Al hacerlo en condiciones de clandestinidad, estas mujeres han corrido el riesgo de contraer infecciones graves y sufrir consecuencias peores, como perder la vida. Mientras tanto, el responsable de esa situación sigue con su vida impunemente.

En este punto, quisiéramos reflexionar acerca de las responsabilidades de cada quién. Ningún juez tiene que recordarles a las mujeres que cumplan con sus obligaciones en torno a la maternidad; es uno de los estereotipos con los que las niñas crecen: que su rol en el mundo es ser madres y asegurar la reproducción de la especie humana. Caso contrario: los hombres frecuentemente necesitan recibir una ordenanza legal para cumplir con sus obligaciones de paternidad porque, de manera voluntaria, prefieren dar la espalda y dejar las labores de crianza, cuidado y manutención de sus descendientes a la mujer.

En el fondo de esta situación, en la falta de reconocimiento a los derechos de las mujeres, se encuentra la terrible misoginia que el Estado ejerce al criminalizar la potestad de la mujer sobre su cuerpo; de ese modo, le impone la carga social de maternar, lo quiera o no. Estas acciones, antes que reconocer a la mujer como sujeta de derechos al mismo nivel que el hombre, están encaminadas a satisfacer a los grupos antiderechos. 
Despenalizar el aborto significa brindarles justicia reproductiva a las mujeres que necesiten hacerlo y a aquéllas que en su momento se vieron obligadas a ser madres. Es una deuda histórica del Estado mexicano y del gobierno de Tabasco hacia nosotras por todos esos atropellos a los derechos humanos de las mujeres, como el derecho a la dignidad, al libre desarrollo de la personalidad, a no vivir discriminación, a la libre manifestación de las ideas y al acceso a una vida libre de violencia.

Hablar de justicia reproductiva significa que se nos reconozca como mujeres autónomas, que podamos decidir en la esfera privada sobre nuestra maternidad de manera libre, responsable e informada, como lo establece el artículo 4 de la Constitución Política de los Estados Unidos Mexicanos (Cámara de Diputados H. Congreso de la Unión, 2021). A las mujeres se nos debe proporcionar toda la información necesaria para acceder a un aborto voluntario y ésta no debe estar sesgada por creencias personales o juicios dogmáticos religiosos; por el contrario, los servicios públicos de salud deben proporcionar estos datos sin intentar persuadir a la mujer de que decida según los prejuicios y no a partir de su propia voluntad.

En cuanto al personal de salud que alega objeción de conciencia para negarse a proporcionar el servicio de aborto, se ha justificado invocando el artículo 10 bis de la Ley General de Salud. ${ }^{17}$ La objeción de conciencia se define como "la negativa de una persona de cumplir con un mandato jurídico, al considerarlo incompatible con sus convicciones fundamentales" (Cancino et al., 2019, p. 8). Resulta irónico que sólo se argumente una objeción cuando se trata de brindar un servicio de salud para interrumpir un embarazo, pero no lo apliquen en casos en los que deben atender a hombres que han dañado seriamente a la sociedad, como los pedófilos, los violadores, los asesinos. Nunca se da ese caso. Entonces, no se trata de objeción de conciencia, sino de prejuicios misóginos que revictimizan a las mujeres.

17 El pasado 20 de septiembre de 2020, la SCJN, mediante una sentencia de acción de inconstitucionalidad, declaró inválido este artículo por contravenir el ejercicio de derechos de terceras personas. 


\section{Conclusión}

¿Cómo podríamos lograr la descriminalización del aborto ante este panorama?

Las feministas proponemos la reforma a los artículos 130, 131, 132, 133, 134, 135 y 136 del Código Penal del Estado de Tabasco para que se despenalice el aborto hasta las doce semanas de gestación, homologando así la legislación local con los códigos de Oaxaca, Ciudad de México, Hidalgo y Veracruz.

Proponemos reformar también la Ley de Salud del estado para asegurar el acceso a un aborto libre y seguro en los hospitales y clínicas públicas y privadas; ello eliminaría las brechas económicas que revictimizan a las mujeres en situación de pobreza.

La reforma contemplaría a la Secretaría de Educación para que se instituya un programa de educación sexual integral con perspectiva de género, en todos los niveles educativos, que se coloque por encima de los prejuicios y las doctrinas religiosas y que sea de observancia obligatoria, aun en los colegios particulares tutelados por religiosas y religiosos.

Porque las mujeres decidimos y el Estado garantiza. ¡Educación sexual para decidir, anticonceptivos para disfrutar, aborto legal para no morir!

\section{Referencias bibliográficas}

Ballinas, J. (2014). Aspiración manual endouterina vs legrado uterino instrumentado en pacientes con diagnóstico de aborto, Hospital General Jilotepec, I.S.E.M. [Tesis de especialidad]. Universidad Autónoma del Estado de México. http://ri.uaemex.mx/bitstream/ handle/20.500.11799/14989/Tesis.420064.pdf

Cámara de Diputados H. Congreso de la Unión (2021, 28 de mayo). Constitución Política de los Estados Unidos Mexicanos. Diario Oficial de la Federación. http://www.diputados.gob.mx/LeyesBiblio/pdf_mov/ Constitucion_Politica.pdf

Cancino, M., Capdevielle, P., Gascón, A. y Medina, M. de J. (2019). Objeción de conciencia. Enseñanza transversal en Bioética y Bioderecho. Cuaderno de casos. Instituto de Investigaciones Jurídicas, Universidad Nacional Autónoma de México. https://archivos.juridicas.unam.mx/www/bjv/ libros/13/6010/5a.pdf

Cano, G. (1990). Una perspectiva del aborto en los años treinta: La propuesta marxista $=$ la propuesta del aborto de la CTM en 1936. Debate Femi- 
nista, 2, 362-372. https://doi.org/https://doi.org/10.22201/ cieg.2594066xe.1990.2.1935

Cano, G. (1988). Memorias del olvido: La despenalización del aborto hace 50 años. Fem, 12(65), 22-23. http://132.248.160.2:8991/pdf_ cih01/000002318

Congreso del Estado de Veracruz (2021, 20 de julio). Dictamen con proyecto de Decreto. Gaceta Legislativa, 3(137). https://www.legisver.gob.mx/ gaceta/gacetaLXV/GACETA137.pdf

Consorcio Latinoamericano Contra el Aborto Inseguro (s. f.). Para abortos seguros confía en el misoprostol. Guía de uso. CLACAI. https://clacaidigital.info/ bitstream/handle/123456789/268/Clacai_miso_folleto2012.pdf

Federación Internacional de Ginecología y Obstetricia (2017). Misoprostol solo: Regímenes recomendados 2017. FIGO. https://www.figo.org/ sites/default/files/2020-06/FIGO\%20Dosage\%20Chart\%20\%20 -\%20SPANISH.pdf

Fondo María (2018). Aborto con medicamentos, una opción segura y eficaz para las mujeres. https://www.fondomaria.org/paginas/ aborto-medicamentos

Ginecea (2021). Legislación del aborto en Hidalgo en 2021. Clínica Gineca. https://clinicaginecea.com.mx/aborto-en-hidalgo-legislacion-en-2021/

Guerra, H. (2019). El acompañamiento feminista durante el proceso de aborto [Tesis de maestría]. Universidad de Ciencias y Artes de Chiapas. https:// repositorio.unicach.mx/bitstream/handle/20.500.12753/868/868.pdf

Instituto Nacional de Estadística y Geografía (2020a). Tasa de desocupación total trimestral según entidad federativa. https://www.inegi.org.mx/ app/tabulados/default.html?nc $=624$

Instituto Nacional de Estadística y Geografía (2020b). Viviendas particulares habitadas por entidad federativa según disponibilidad de bienes, años censales de 2000, 2010 y 2020. https://www.inegi.org.mx/ app/tabulados/interactivos/?pxq=Vivienda_Vivienda_05_210ad0cf-6644-4ad9-a6a6-08341920229c\&idrt $=56 \&$ opc $=\mathrm{t}$

Lagarde, M. (2006). Pacto entre mujeres. Sororidad. Aportes para el Debate, 25, 123-135. https://www.asociacionag.org.ar/pdfaportes/25/09.pdf

Lerner, G. (1986). La creación del patriarcado. Crítica.

Major, B., Appelbaum, M., Beckman, L., Dutton, M. A., Russo, N. F. y West, C. (2009). Abortion and mental health. Evaluating the evidence. American Psychologist, 64(9), 863-890. 
Morales, A. (2019). Acompañar el aborto. Letra S. (252). https://letraese. jornada.com.mx/2019/09/04/acompanar-el-aborto-5093.html

Olvera, J., Villavicencio, Y., Alfie, S., Rocha, M., Ruiz, M. y Hernández, C. (2021, 2 de mayo). Acompañantas de aborto: Entre la solidaridad y la criminalización. Corriente Alterna. https://corrientealterna.unam.mx/genero/ acompanantas-de-aborto-entre-la-solidaridad-y-la-criminalizacion/

Organización Mundial de la Salud (2014). Manual de práctica clínica para un aborto seguro. https://www.who.int/reproductivehealth/publications/unsafe_abortion/clinical-practice-safe-abortion/es/

Poder Ejecutivo del Estado de Hidalgo (2021, 6 de julio). Decreto 728, que reforma los artículos 154, 155, 156 y 158, y deroga el 157 del Código Penal para el Estado de Hidalgo. Periódico Oficial del Estado de Hidalgo. https://periodico.hidalgo.gob.mx/?p=65523

Secretaría de Salud (2009, 16 de abril). NOM-046-SSA2-2005, Violencia familiar, sexual y contra las mujeres. Criterios para la prevención y atención. Diario Oficial de la Federación. https://www.gob.mx/ salud/cnegsr/documentos/norma-046-en-el-dof

Secretariado Ejecutivo del Sistema de Seguridad Pública (2021, 31 de julio). Incidencia delictiva del fuero común. Instrumento para el Registro, Clasificación y Reporte de Delitos y las Víctimas. CNSP/38/15. https:// drive.google.com/file/d/1JuDUt3rphLlmT4h-J66w-mMkiw5bEsJw/view

Senado de la República (2011, 10 de agosto). Iniciativa con proyecto de Decreto. Gaceta de la Comisión Permanente del Senado de la República. https://www.senado.gob.mx/64/gaceta_comision_permanente/documento/31245

Subsistema Automatizado de Egresos Hospitalarios (2021). Formatos de Egresos 2021. http://www.dgis.salud.gob.mx/contenidos/sinais/s_saeh.html

Tribunal Superior de Justicia de Tabasco (2020). ¿Cuántas mujeres están cumpliendo y cuántas mujeres cumplieron sentencia por los delitos de aborto, homicidio en razón de parentesco o infanticidio en el Estado de Tabasco desde el año 2000 hasta la fecha de esta solicitud? [Solicitud de acceso a la información pública presentada ante el Instituto Nacional de Transparencia, Acceso a la Información y Protección de Datos Personales]. Infomex. 